\title{
In Vitro and In Silico ADME-Tox Profiling and Safety Significance of Multifunctional Monoamine Oxidase Inhibitors Targeting Neurodegenerative Diseases
}

Anna Więckowska,* Natalia Szałaj, Izabella Góral, Adam Bucki, Gniewomir Latacz, Katarzyna Kiec-Kononowicz, Òscar. M. Bautista-Aguilera, Alejandro Romero, Eva Ramos, Javier Egea, Victor Farré Alíns, Águeda González-Rodríguez, Francisco López-Muñoz, Mourad Chioua, and José Marco-Contelles

Cite This: ACS Chem. Neurosci. 2020, 11, 3793-3801

Read Online

ACCESS

Wl Metrics \& More

Article Recommendations

Supporting Information

ABSTRACT: Herein we report in vitro metabolic stability in human liver microsomes (HLMs), interactions with cytochrome P450 isoenzymes (CYP3A4, CYP2D6, and CYP2C9), and cytotoxicity analyses on HEK-293, HepG2, Huh7, and WTIIB cell lines of our most recent multitarget directed ligands PF9601N, ASS234, and contilisant. Based on these results, we conclude that (1) PF9601N and contilisant are metabolically stable in the HLM assay, in contrast to the very unstable ASS234; (2) CYP3A4 activity was decreased by PF9601N at all the tested concentrations and by ASS234 and contilisant only at the highest concentration; CYP2D6 activity was reduced by ASS234 at 1, 10, and $25 \mu \mathrm{M}$ and
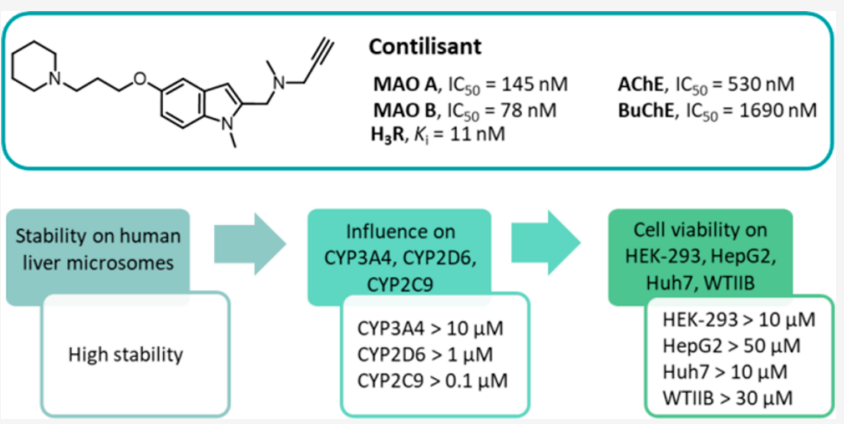
by PF9601N at 10 and $25 \mu \mathrm{M}$, whereas contilisant increased its activity at the same concentrations; CYP2C9 was inhibited by the three compounds; (3) contilisant did not affect cell viability in the widest range of concentrations: up to $10 \mu \mathrm{M}$ on HEK-293 cells, up to $30 \mu \mathrm{M}$ on Huh7 cells, up to $50 \mu \mathrm{M}$ on HepG2 cells, and up to 30 or $100 \mu \mathrm{M}$ on WTIIB cells. Based on these results, we selected contilisant as a metabolically stable and nontoxic lead compound for further studies in Alzheimer's disease therapy.

KEYWORDS: ADME-tox, Alzheimer's disease, ASS234, Contilisant, Cytochrome P450 enzymes, Cytotoxicity, MAO inhibitors, Metabolism, Multitarget directed ligands, PF9601N

\section{INTRODUCTION}

Neurodegenerative diseases, such as Parkinson's disease (PD) and Alzheimer's disease (AD), are characterized by a complex pathogenesis that is the main cause of the difficulties found in their effective treatment. An age-dependent progressive loss of different populations of neurons, dopaminergic in PD and cholinergic in $\mathrm{AD}$, together with protein misfolding, oxidative stress and neuroinflammation, lead to movement disturbances in $\mathrm{PD}$ and cognitive impairment in $\mathrm{AD}$, that are accompanied by behavioral and psychological disorders. ${ }^{1-3}$ Currently approved treatment strategies in $\mathrm{PD}$ and $\mathrm{AD}$ are based mainly on enhancing the level of neurotransmitters that are lowered due to neuronal cell death. ${ }^{4}$ These approaches temporarily delay and reduce the symptoms but cannot stop the progression of neurodegeneration and disease development. Thus, the main attempts in drug discovery are directed at search for disease-modifying therapeutics. Also, due to the complexity of neurodegenerative disorders, the focus is on multitarget-directed ligands (MTDLs). MTDLs act on multiple, carefully selected biological targets and therefore may modify several pathological processes involved in the pathogenesis of the disease. ${ }^{5}$

PF9601N, ASS234, and contilisant are indole- and $\mathrm{N}$ propargylamine-based multifunctional ligands developed in Laboratory of Medicinal Chemistry in Madrid, Spain (Figure 1). ${ }^{6-9}$ They combine monoamine oxidase (MAO) inhibitory potency with other biological activities relevant for the treatment of PD and AD. Compound PF9601N is a dopamine uptake inhibitor with neuroprotective effects demonstrated in several cellular and in vivo models of PD. ${ }^{10,11}$ ASS234 inhibits human cholinesterases $\left(h \mathrm{AChE} \mathrm{IC}_{50}=810 \mathrm{nM}, h \mathrm{BuChE} \mathrm{IC}_{50}\right.$ $=1820 \mathrm{nM})$, self-induced and AChE-induced $\mathrm{A} \beta$ aggregation

Received: July 29, 2020

Accepted: October 21, 2020

Published: November 3, 2020 

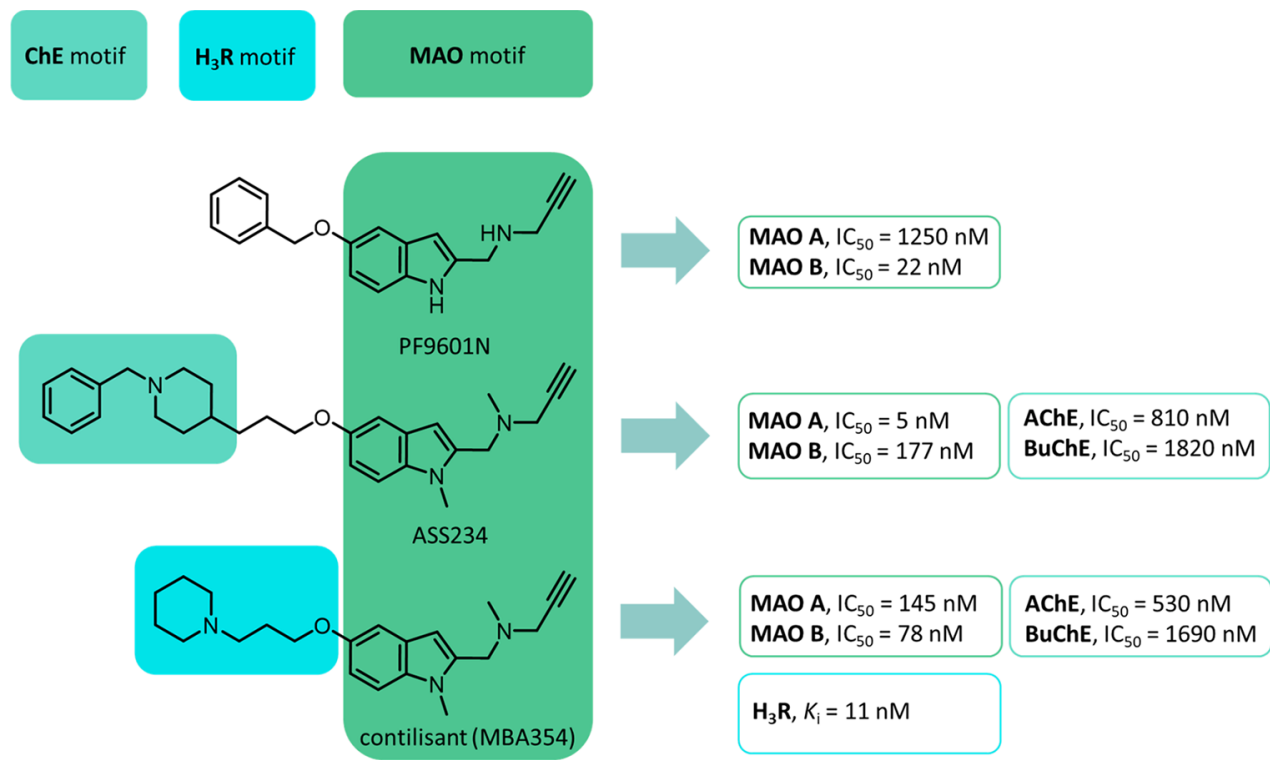

$\mathrm{H}_{3} \mathrm{R}, \mathrm{K}_{\mathrm{i}}=11 \mathrm{nM}$

Figure 1. Multifunctional MAO inhibitors as anti-Parkinson's and anti-Alzheimer's agents.

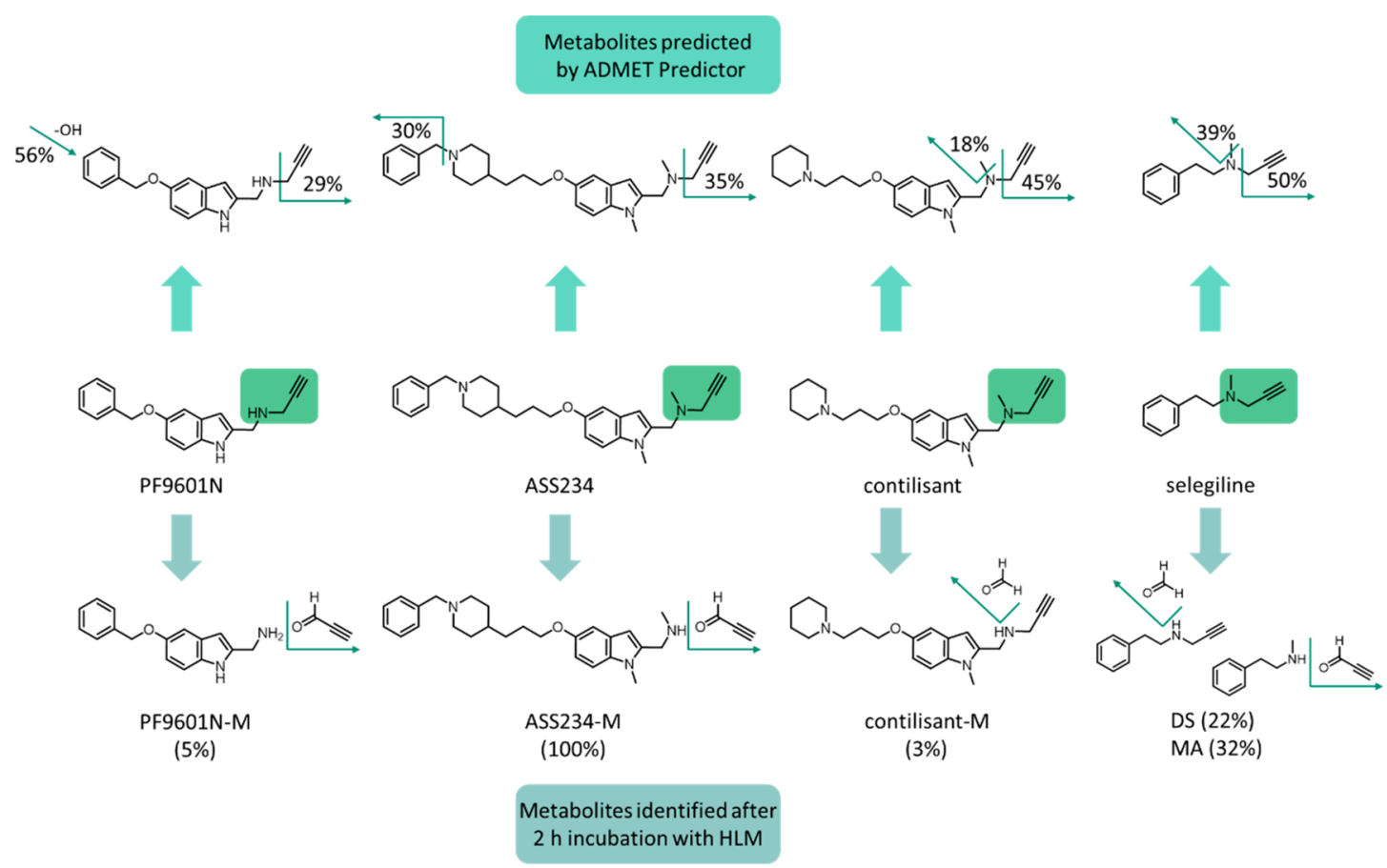

Figure 2. Major metabolic pathways predicted in ADMET Predictor and metabolites PF9610N-M, ASS234-M, contilisant-M, desmethylselegiline (DS), and methamphetamine (MA) detected after incubation of PF9601N, ASS234, contilisant, and selegiline with HLMs.

and activates sigma- 1 receptors $\left(K_{\mathrm{i}} h \mathrm{~S} 1 \mathrm{R}=3 \mathrm{nM}\right) .^{7}$ It also shows a protective effect against $\mathrm{A} \beta_{1-42}$-induced toxicity and depletion of antioxidant enzymes in SH-SY5Y human neuroblastoma cells. ${ }^{12}$ Further ex vivo and in vivo studies showed that ASS234 reduces the $\mathrm{A} \beta$ plaque burden and neuroinflammation in the mouse cortex and displays significant improvements in memory processes in a scopolamine-induced cognitive deficit test, comparable with donepezil. ${ }^{7}$ Contilisant is the most advanced preclinical candidate for the treatment of $\mathrm{AD}$ (Figure 1). ${ }^{8,9}$ Contilisant, like ASS234, also shows activity toward cholinesterases $\left(h \mathrm{AChE} \mathrm{IC} \mathrm{I}_{50}=530 \mathrm{nM}, h \mathrm{BuChE} \mathrm{IC}_{50}=1690\right.$ $\mathrm{nm})$ and is a sigma- 1 receptor agonist $\left(K_{\mathrm{i}} h \mathrm{~S} 1 \mathrm{R}=65 \mathrm{nM}\right)$, but it also shows high activity toward the histamine $\mathrm{H} 3$ receptor
$\left(\mathrm{H} 3 \mathrm{R} K_{\mathrm{i}}=11 \mathrm{nM}\right)$. A multidirectional in vitro profile of contilisant activity is reflected in its exceptional in cellulo and in vivo profile. Contilisant protected SH-SY5Y human neuroblastoma cells against damages caused by ROS generators (rotenone and oligomycin $\mathrm{A}$ ), $\mathrm{A} \beta_{25-35}$ and hyperphosphorylation of proteins caused by okadaic acid. Contilisant was shown to restore cognitive impairment induced by $\mathrm{A} \beta$ oligomers in Y-maze and radial maze assays and by lipopolysaccharide in the novel object recognition test. To sum up, PF9601N, ASS234, and contilisant are all interesting compounds in terms of their biological properties and potential utility in the treatment of neurodegenerative diseases such as $\mathrm{PD}$ or $\mathrm{AD}$. 
A
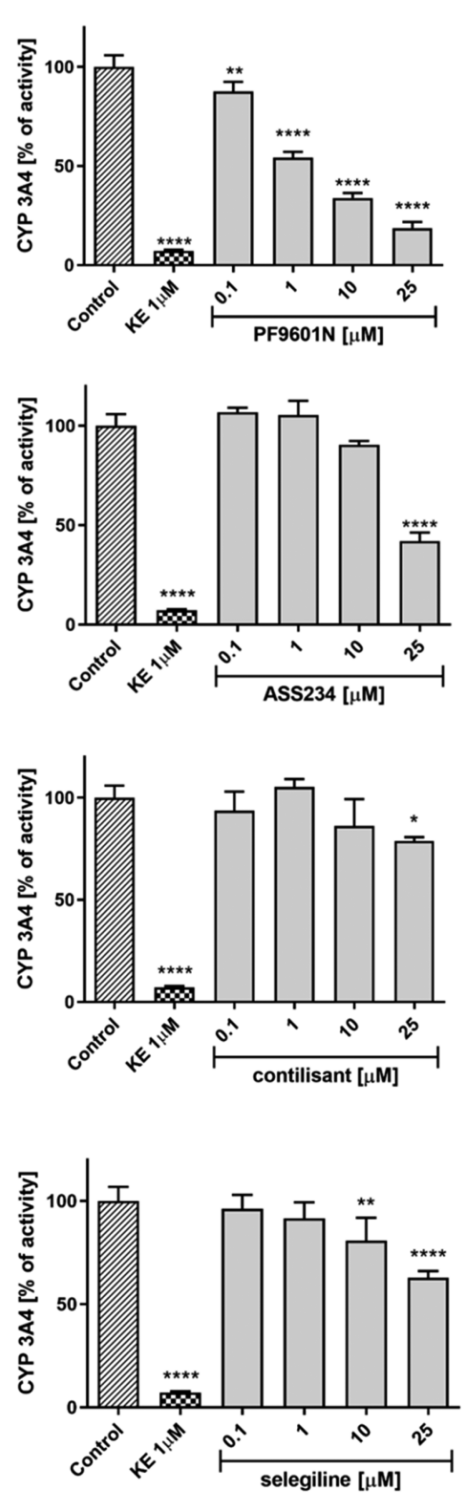

B
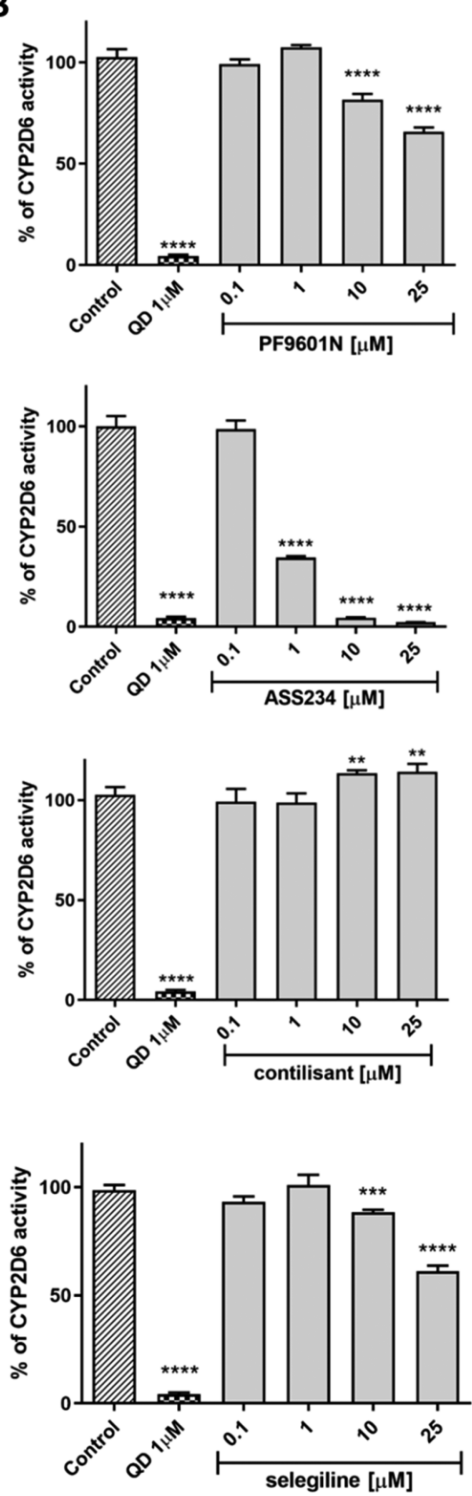
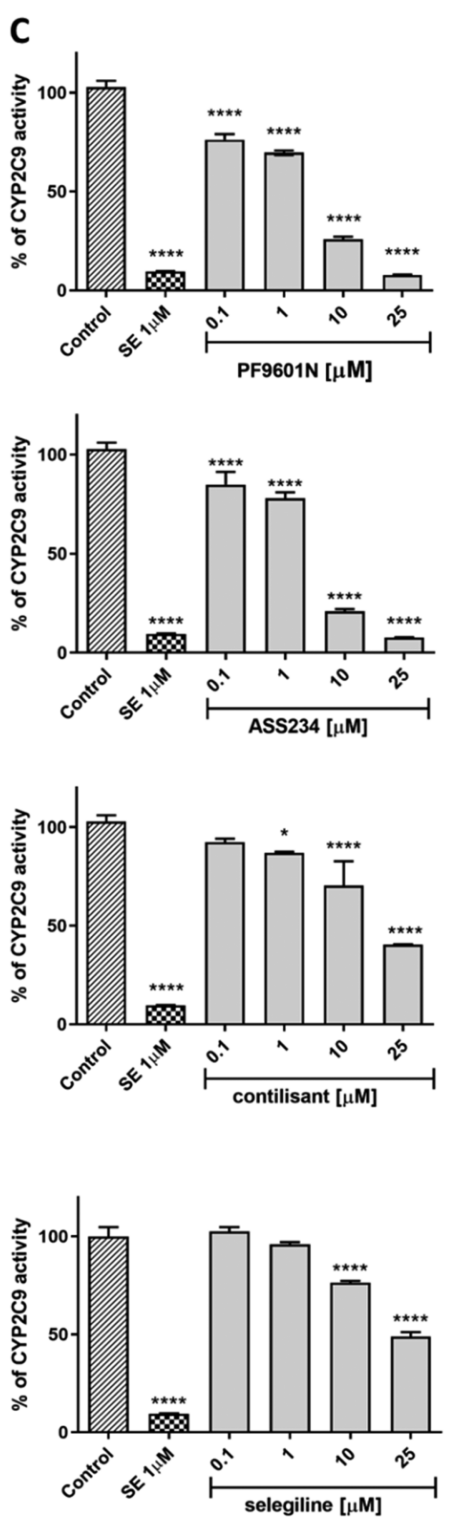

Figure 3. Influence of PF9601N, ASS234, contilisant and selegiline on (A) CYP3A4 activity, (B) CYP2D6 activity, and (C) CYP2C9 activity. Statistical significance was evaluated by one-way ANOVA, followed by Bonferroni's comparison test $(* p<0.05, * * p<0.01, * * * * p<0.0001)$. KE, ketoconazole; QD, quinidine; SE, sulfaphenazole.

In the drug discovery process, equally important to the biological activity of the compounds is their drug-likeness. It arises from structural (e.g., molecular weight, polar surface area, and hydrogen bond donor/acceptor count), physicochemical (e.g., solubility and chemical stability), or biochemical (e.g., metabolic stability, selectivity, and off-target activity) parameters that determine the absorption, distribution, metabolism, excretion, and toxicity (ADME-tox) profile of each compound. ${ }^{13}$ These parameters should be considered and optimized from the very beginning of the drug development process. Herein, we focus on the selected biochemical parameters (metabolic stability) and safety issues and present a preliminary in vitro evaluation of metabolic stability in human liver microsomes and cytochrome P450 isoenzymes inhibition and cytotoxicity on HEK-293, HepG2, Huh7, and WTIIB cell lines of the known MTDLs, PF9601N, ASS234, and contilisant (Figure 1), targeting processes relevant to $\mathrm{PD}$ and $\mathrm{AD} .^{6-9}$

\section{RESULTS AND DISCUSSION}

2.1. Metabolic Stability. The metabolic stability of a compound is an important factor that determines its pharmacokinetics and its potential as a drug candidate. Drugs are being metabolized in a variety of tissues including lungs, kidneys, or intestinal walls, but the liver plays an essential role in this process. Therefore, we have analyzed the metabolic stability of PF9601N, ASS234, and contilisant in vitro on human liver microsomes (HLMs). For comparison in these studies, we have used selegiline, a marketed MAO inhibitor which shares structural similarity with the tested compounds. We have incubated the compounds with HLMs for $2 \mathrm{~h}$ and analyzed the mixture with UPLC-MS, according to the previously reported method (for details considering these experiments, including UPLC-MS spectra, see the Supporting Information). ${ }^{14}$ The common structural fragment in all the compounds discussed herein, that is susceptible to metabolism, in principle oxidative $\mathrm{N}$-dealkylation, is $\mathrm{N}$-(methyl)propargylamine. The experimental data showed that the 


\section{A}
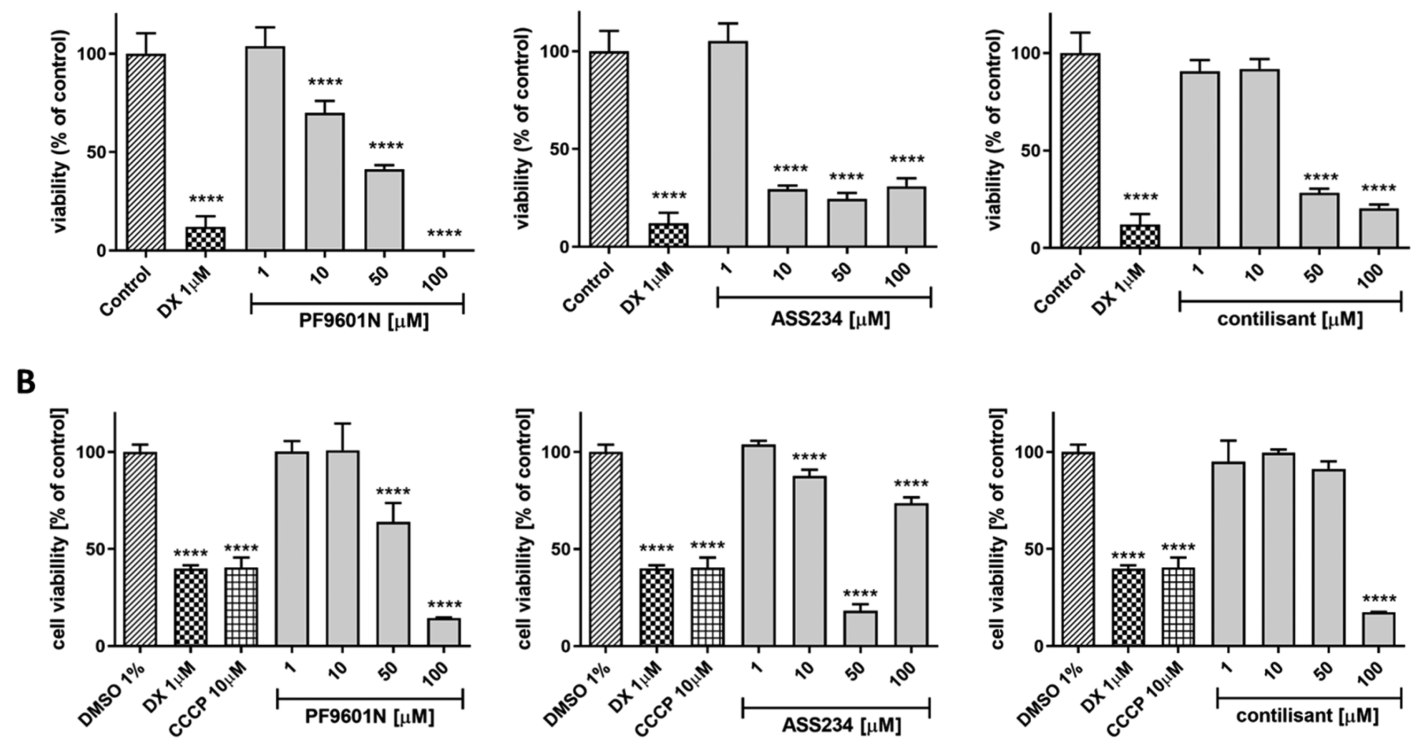

Figure 4. Cell viability measured in MTS assay on HEK-293 and HepG2 cell lines after incubation with PF9601N, ASS234, and contilisant for 72 h. (A) Concentration-response on HEK-293 cells. (B) Concentration-response on HepG2 cells. Statistical significance was evaluated by one-way ANOVA, followed by Bonferroni's comparison test $(* * * *<0.0001)$. DX, doxorubicin; CCCP, carbonyl cyanide 3-chlorophenyl hydrazine. ASS234 precipitated at $100 \mu \mathrm{M}$ during incubation.

compounds are metabolized by $\mathrm{N}$-demethylation (contilisant, selegiline) and N-depropargylation (PF9601N, ASS234, selegiline) (Figure 2). This is indicated by molecular masses decreased by 14 and by 38 , corresponding the masses of methyl and propargyl groups, respectively. Since there are two spots where demethylation is possible in contilisant, we have performed fragmentation analysis. It showed that the methyl group in $\mathrm{N}$-(methyl)propargylamine is removed. We were happy to find that our experimental data are in line with the metabolic pathways predicted by the ADMET Predictor tool. For all the compounds the program predicted oxidative $\mathrm{N}$ dealkylation as one of two major metabolic pathways (Figure 2). According to the predictions, PF $9601 \mathrm{~N}$ is more susceptible to para-hydroxylation of the benzyl ring, but this metabolite did not occur in vitro. Considering the structural similarity of the compounds, it was interesting to find out that while PF9601N and contilisant are metabolically stable in vitro (95\% and $97 \%$ of the parent compounds respectively were detected), selegiline is metabolized in about $54 \%$ and ASS234 was greatly unstable (the compound was not detected). After a $2 \mathrm{~h}$ incubation with ASS234, only its metabolite ASS234-M was detected (Figure 2). Although it was quite surprising, these results were confirmed by the predicted clearance of the compounds, that was the lowest for contilisant $(82.1 \mu \mathrm{L} / \mathrm{min} /$ mg HLM protein), higher for PF9601N (140.1 $\mu \mathrm{L} / \mathrm{min} / \mathrm{mg}$ HLM protein), and very high for ASS234 (358.8 $\mu \mathrm{L} / \mathrm{min} / \mathrm{mg}$ HLM protein).

2.2. Influence on Cytochrome P450 (CYPs) Isoenzymes Activity. Cytochrome P450 enzymes (CYPs) are responsible for the liver metabolic transformation of the majority of drugs; therefore ,they are also sites of drug-drug interactions. Inhibition or induction of CYPs by one drug may lead to the accumulation, potential toxicity, or intensified metabolism and therefore the lack of activity of the other one. Additionally, the reactive terminal acetylene functionality in MAO inhibitors not only determines their mechanism of action but also is associated with mechanism-based inactivation of CYPs. ${ }^{15}$ Consequently, assessing the impact of these compounds on CYP activity is a crucial step in their development. We tested the influence of PF9601N, ASS234, contilisant, and selegiline on the activity of the most common isoforms of Cytochrome P450, CYP3A4, CYP2D6, and CYP2C9, and compared them with well-known inhibitors, such as ketoconazole, quinidine, and sulfaphenazole, respectively (Figure 3). CYP3A4 activity was decreased by PF9601N at all the tested concentrations $(0.1,1,10$, and $25 \mu \mathrm{M})$, by selegiline at 10 and $25 \mu \mathrm{M}$, and by ASS234 and contilisant only at the highest concentration of $25 \mu \mathrm{M}$ (Figure 3A). Regarding the influence on CYP2D6 activity, the most potent inhibitor of this enzyme was ASS234, which decreased enzyme activity at 1,10 , and $25 \mu \mathrm{M}$. Also, PF9601N and selegiline decreased CYP2D6 activity at 10 and $25 \mu \mathrm{M}$, but contilisant, on the other hand, seemed to increase the activity of the enzyme at the same concentrations. Finally, we found that all the compounds inhibited CYP2C9. It should be noted that the inhibitory activities of the tested compounds against CYP3A4, CYP2D6, and CYP2C9 were lower than the activities of ketoconazole, quinidine, and sulfaphenazole used here as references. The impact of contilisant and selegiline on CYP activity was observed only at the highest tested concentrations (10 and 25 $\mu \mathrm{M})$.

2.3. Cytotoxicity on Kidney HEK-293 and Liver HepG2, Huh7, and WTIIB Cell Lines. Safety is among the most important issues in the drug discovery process and, at the same time, one of the major factors leading to drug failures. High throughput in vitro cytotoxicity studies are wellestablished methods that allow the early detection and elimination of compounds with toxicity issues from further development. In our studies, we determined the preliminary safety of the compounds by measuring the viability of cells exposed to these compounds. Due to some discrepancies ${ }^{16}$ that have been found when various methods used to measure cell viability were compared, we used three different assays: MTS, MTT that indirectly indicates the number of viable cells, 

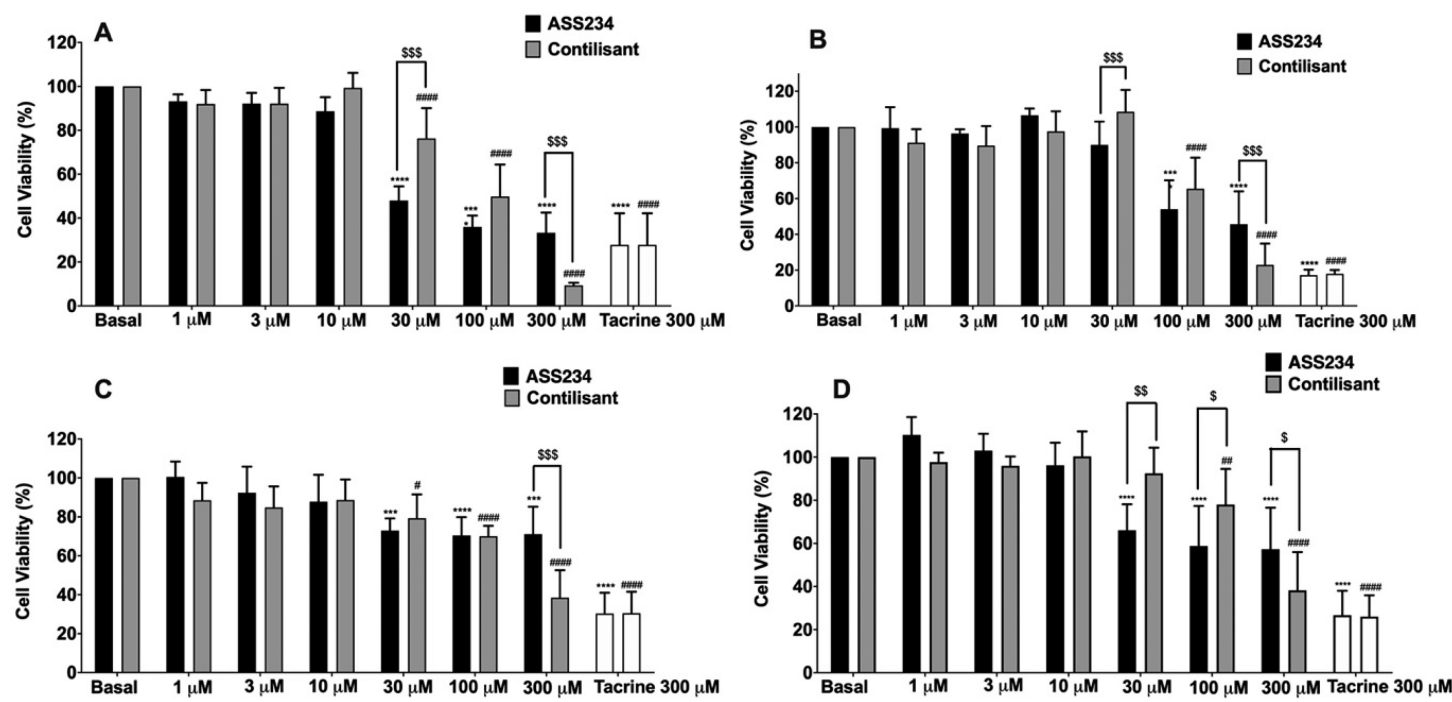

Figure 5. Cell viability measured in the MTT and crystal violet assays on Huh7 and WTIIB cell lines. Experiments were run in parallel using contilisant, ASS234, and tacrine $(300 \mu \mathrm{M})$. (A) Concentration-response on Huh7 cells measured by MTT assay after $24 \mathrm{~h}$ incubation with compounds. (B) Concentration-response on WTIIB cells measured by MTT assay after $24 \mathrm{~h}$ incubation with compounds. (C) Concentrationresponse on Huh7 cells measured by crystal violet assay after $24 \mathrm{~h}$ incubation with compounds. (D) Concentration-response on WTIIB cells measured by crystal violet assay after $24 \mathrm{~h}$ incubation with compounds. Data correspond to the mean \pm SEM of at least six different cell batches in triplicate. $* * * p<0.0001$ and $* * * p<0.001$ with respect to ASS234 and basal nontreated groups. ${ }^{* \# \#} p<0.0001,{ }^{\# \#} p<0.01$, and ${ }^{\#} p<0.05$ comparing contilisant with basal nontreated groups. ${ }^{\$ \$} p<0.001,{ }^{\$ \$} p<0.01$, and ${ }^{\$} p<0.05$ comparing contilisant with ASS234 treated groups.

and crystal violet assay (CV), a direct method in which the DNA mass of living cells is measured (Figure 4). ${ }^{17}$ We used cell lines of different origin: human kidney HEK-293 and human hepatic HepG2 cell lines that are commonly used in drug metabolism and cytotoxicity studies. To extend the cytotoxicity profile, we additionally used human hepatic Huh7 and murine WTIIB cell lines. ASS234 was previously tested in HepG2 cells, ${ }^{18}$ but to further test its safety and to compare it with two other compounds of interest, we included it in the studies. First, all of the compounds were tested on HEK-293 and HepG2 cell lines in the MTS test. None of the compounds decreased any cell line viability at the concentration of $1 \mu \mathrm{M}$. Among the tested compounds, contilisant displayed the most promising profile. It did not affect cell viability in the widest range of concentrations, up to $10 \mu \mathrm{M}$ on HEK-293 and up to $50 \mu \mathrm{M}$ on HepG2 cells.

Additionally, ASS234 and contilisant were tested in a wider range of concentrations $(1-300 \mu \mathrm{M})$ in two assays, MTT and $\mathrm{CV}$, on two more cell lines, human hepatic Huh7 and murine hepatic WTIIB. We obtained similar results independently of the hepatic cell line and the viability assay used. From $30 \mu \mathrm{M}$ onward, the compounds began to elicit toxicity (Figure 5), except for contilisant in WTIIB cells (Figure 5B and D), which started at $100 \mu \mathrm{M}$ in both assays. We observed that contilisant was less toxic than ASS234 at the lowest toxic concentration (30 $\mu \mathrm{M}$, Figure $5 \mathrm{~A}$, and $\mathrm{C}$ ). Furthermore, contilisant was less toxic than ASS234 at the concentration of $100 \mu \mathrm{M}$ when the $\mathrm{CV}$ assay was used in WTIIB cells (Figure 5D). However, at the highest concentration $(300 \mu \mathrm{M})$, contilisant increased its toxicity and became more toxic than ASS234 (Figure 5A-D) in both cell lines. Nevertheless, this concentration is far higher than the effective concentration of contilisant $(0.3-3 \mu \mathrm{M})$ used in neuroprotection experiments. ${ }^{8}$

2.4. In Silico Toxicity Studies. For more detailed information in terms of safety of contilisant, we performed in silico prediction of the potentially toxic effects of the compound and its metabolites. We performed toxicity and mutagenicity predictions with Derek Nexus that rely on the comparison of the structural features of the tested compound with one or more toxicophore patterns (structural alerts) in Lhasa's Knowledge Base. ${ }^{19}$

In terms of toxicity, from among 59 end points analyzed by Derek Nexus for contilisant, no alerts were fired for 57 (see the Supporting Information); $\alpha$-2- $\mu$-globulin nephropathy in humans was considered impossible, and only hERG channel inhibition in vitro in humans was considered plausible. The blockage of this channel can lead to the lengthening of the ventricular repolarization phase in the heart and is characterized in the electrocardiogram as a prolongation of the QT interval. $^{20}$ As this is a common feature that makes molecules fall out in the preclinical phases, a deeper investigation should be performed for contilisant at this point.

The mutagenicity of contilisant was assessed by two complementary QSAR methods: Derek Nexus (KB 2018 1.1) and Sarah Nexus (KB 2018 1.1). The compound did not match any structural alerts or examples for bacterial in vitro mutagenicity. Additionally, it did not contain any unclassified or misclassified features and is consequently predicted to be inactive in the bacterial in vitro mutagenicity test (Ames test). Furthermore, predicted Phase I metabolites of contilisant were evaluated in terms of toxicity and mutagenic potential (see the Supporting Information for details). Results showed that, out of 15, 7 metabolites were not associated with any probable/ plausible structural toxicity alerts by Derek, 7 were associated with plausible hERG channel inhibition, and 1 was associated with carcinogenic/teratogenic risk. In terms of carcinogenic risk, both Derek (KB 2018 1.1) and Sarah (model 2.0) predicted no structural alerts for contilisant and its 12 metabolites, which under ICH M7 guidelines is sufficient to conclude that there is no mutagenic concern and no further testing is recommended for them (Class 5). However, two metabolites (M3, prop-2-yn-1-ol and M4, propiolic acid) 
obtained an uncertain result. Whereas Derek predicted a confident negative prediction, Sarah gathered a positive or an equivocal prediction. One metabolite fired a positive bacterial mutagenicity result, after finding an exact match to be $100 \%$ positive and overturning the negative result obtained by Derek. Among these metabolites, only contilisant-M (Figure 3) was the one detected in $3 \%$ after $2 \mathrm{~h}$ incubation of contilisant with HLMs, as described above. It was associated with plausible hERG channel inhibition and no other toxicity or mutagenicity alerts.

In addition to the recommended studies, the concept of Threshold of Toxicological Concern (TTC) has been developed to define an acceptable intake for any unstudied chemical that poses a negligible risk of carcinogenicity or other toxic effects. ${ }^{21}$ The exposure to a metabolite identified as a mutagen is not necessarily associated with an increased cancer risk, additional analytical controls should be developed to ensure that the mutagenic impurity is at or below the acceptable cancer risk level. ${ }^{21}$ Despite the outcome detected for $\mathrm{M} 3$, the existence of a potential impurity structural alert alone is considered insufficient to assess carcinogenicity risk. Considering TTC-based acceptable intake of mutagenic impurity (1.5 $\mu \mathrm{g}$ person/day) can derive an acceptable limit for control for long-term treatments. Indeed, the only metabolite identified after HLMs incubation of contilisant was contilisant-M, and only in $3 \%$.

\section{CONCLUSIONS}

In this work, we have analyzed the metabolism, the inhibition of cytochrome P450 isoenzymes activity, and the cytotoxicity of PF9601N, ASS234, and contilisant, selected MTDLs discovered in Laboratory of Medicinal Chemistry in Madrid as potential therapeutic agents for neurodegenerative diseases, such as $\mathrm{AD}$ and $\mathrm{PD}$. These analyses would eventually provide key pieces of information to support potential agent in preclinical phases.

Regarding their metabolism, we have observed that the compounds are metabolized by $\mathrm{N}$-demethylation (contilisant) and N-depropargylation (PF9601N, ASS234). Although they have a similar pattern of metabolism in HLMs, which is due to the structural similarities, they differ in terms of their stability on HLMs and the predicted clearance. Very interestingly, while ASS234 was greatly unstable, PF9601N and contilisant were metabolically stable, results that were also confirmed in the predicted clearance of the compounds, with the lowest for contilisant, higher for PF9601N, and very high for ASS234. This is a very important issue that guarantees a longer sustained therapeutic effect at a much lower dose administration. It is worth mentioning that in these studies selegiline, currently used in PD therapy, ranks between contilisant and ASS234 which gives a definite advantage to contilisant.

Considering the inhibition of cytochrome P450 isoenzymes activity, we tested the influence of PF9601N, ASS234, and contilisant on the activity of CYP3A4, CYP2D6, and CYP2C9. The inhibitory activities of the tested compounds against these CYPs were lower than the activity of ketoconazole, quinidine, and sulfaphenazole, the references used in this study. Contilisant showed the most favorable profile when it comes to interactions with CYPs, and its activity was similar to that of selegiline.

Considering cytotoxicity, we have concluded that none of the molecules decreased HEK-293 and HepG2 cell line viability at the concentration of $1 \mu \mathrm{M}$ and contilisant displayed the most promising profile as it did not affect cell viability in the widest range of concentrations also in Huh7 and WTIIB cell lines. These concentrations were significantly higher than the effective concentration of contilisant $(0.3-3 \mu \mathrm{M})$ used in neuroprotection cellular experiments.

Finally, according to Derek Nexus and Sarah Nexus predictions, contilisant as well as its metabolites identified in vitro do not fire mutagenicity alerts and it can be categorized in ICH M7 as Class 5. In terms of toxicity, two positive or equivocal results were obtained, which means that further hazard assessment or control measures are needed (Class 2). First, further metabolism studies should be developed to confirm whether these metabolites really appear, and afterward, the levels of these possible metabolites should be described.

To sum up, based on the precedent in vitro and in vivo studies, ${ }^{8,9}$ coupled to the present ADME-tox studies, we have identified the most attractive agent among our hit-agents PF9601N, ASS234, and contilisant. Thus, MTDL contilisant is metabolically stable, able to inhibit CYP3A4 only at $25 \mu \mathrm{M}$, nontoxic, patented, ${ }^{22}$ and ready to be launched as a lead compound in a preclinical program for $\mathrm{AD}$ therapy.

\section{METHODS}

ADME-tox parameters of PF9601N, ASS234, contilisant, and selegiline were performed according to the previously described assays and protocols. ${ }^{14,17,23}$ Statistical significances were analyzed by GraphPad Prism 8.0 or GraphPad Prism 6.0 for Mac OS X using oneway ANOVA followed by Bonferroni's multiple comparison post test, Newman-Keuls posthoc test, and Sidak's posthoc test when appropriate. Differences were considered to be statistically significant when $p \leq 0.05$.

4.1. Metabolic Stability. The metabolic pathway determination in vitro was performed by $120 \mathrm{~min}$ incubation of PF9601N, ASS234, contilisant, and selegiline with HLMs (Sigma-Aldrich, St. Louis, MO, USA $)$ in $10 \mathrm{mM}$ Tris- $\mathrm{HCl}$ buffer $(\mathrm{pH}=7.4)$ at $37^{\circ} \mathrm{C}$ in the presence of the NADPH Regeneration System (Promega, Madison, WI, USA). Then, the UPLC/MS analyses were done by using a Waters ACQUITY TQD system with the TQ Detector (Waters, Milford, MA, USA). The in silico prediction of the most probable sites of metabolism was done by ADMET Predictor, ver. 9.5.0.16, Lancaster, CA, USA: Simulations Plus, Inc.; 2019, licensed for Jagiellonian University Medical College.

4.2. Influence on Cytochrome P450 (CYPs) Isoenzymes Activity. The potential drug-drug interactions were predicted using CYP3A4, CYP2D6, and CYP2C9 P450-Glo assays purchased from Promega (Madison, WI, USA) according to a manufacturer's protocol. PF9601N, ASS234, contilisant, and selegiline were tested in triplicate at four concentrations $(0.1,1,10$, and $25 \mu \mathrm{M})$. As the reference inhibitors, ketoconazole (KE), quinidine (QD), and sulfaphenazole (SE) were used (Sigma-Aldrich, St. Louis, MO, USA) for CYP3A4, CYP2D6, and CYP2C9, respectively.

4.3. Cytotoxicity on HEK-293 and HepG2 Cell Lines. Cytotoxicity and hepatotoxicity were estimated using the human embryonic kidney HEK-293 cell line (ATCC CRL-1573) and hepatoma HepG2 (ATCC HB-8065) cell line. The CellTiter 96 Aqueous Non-Radioactive Cell Proliferation Assay (MTS) was purchased from Promega (Madison, WI, USA) and performed according to the manufacturer's protocol. The viability of cells was assessed after $72 \mathrm{~h}$ of incubation with PF9601N, ASS234, and contilisant in quadruplicate at four concentrations $(1,10,50$, and 100 $\mu \mathrm{M})$. The antiproliferative drug doxorubicin (DX) $(1 \mu \mathrm{M})$ and mitochondrial toxin carbonyl cyanide 3-chlorophenyl-hydrazone (CCCP) $(10 \mu \mathrm{M}$, only in hepatotoxicity assay) were used as positive controls (purchased from Sigma-Aldrich, St. Louis, MO, USA).

4.4. Quantification of Cell Viability on Huh7 and WTIIB Cell Lines. The human hepatoma cell line (Huh7) was purchased from the American Type Culture Collection (ATCC, Manassas, VA, USA). 
The generation and characterization of the immortalized mouse hepatocyte cell line WTIIB has been previously described. ${ }^{24}$ Cell viability was measured by the quantitative colorimetric MTT (3-[4,5dimethylthiazol-2-yl]-2,5 diphenyl tetrazolium bromide) assay as previously described, and triarylmethane dye (violet) assay following the protocol adapted from Feoktistova et al. ${ }^{25}$ After the incubation period with the compounds, MTT reagent was added to each well at a final concentration of $0.5 \mathrm{mg} / \mathrm{mL}$. The plate was placed in a humidified incubator at $37{ }^{\circ} \mathrm{C}$ with $5 \% \mathrm{CO}_{2}$ and $95 \%$ air for $2 \mathrm{~h}$. Then, the precipitated formazan was dissolved with dimethyl sulfoxide (DMSO) and the colorimetric determination of MTT was measured in the spectrophotometer at $540 \mathrm{~nm}$ wavelength. Nontreated cells were considered controls and were taken as $100 \%$ viability.

To perform the crystal violet assay, wells were washed twice in PBS and cells were fixed with $100 \mu \mathrm{L}$ of crystal violet (Sigma-Aldrich, Madrid, Spain) solution at $0.2 \%$ prepared in ethanol. Plates were incubated for $20 \mathrm{~min}$ at room temperature in a bench rocker. Next, wells were washed four times with distilled water, and then the plate was inverted gently to remove any remaining liquid. The plate was dried at room temperature for at least $2 \mathrm{~h}$ and attached cells were resuspended in SDS $1 \%$ for $30 \mathrm{~min}$ in a bench rocker. The optical density was measured in the spectrophotometer at $540 \mathrm{~nm}$ wavelength. Nontreated cells were considered controls and were taken as $100 \%$ viability.

4.5. In Silico Prediction of Contilisant Toxicity. To assess toxicity prediction, we used Derek Nexus ver. 6.0.1 (Knowledge Base (KB) 2018 1.1, species: human), which is a knowledge-based expert system by Lhasa Limited, where toxicity predictions consider the presence of one or more toxicophore patterns in the query structure and are the result of two processes: evaluating alerts and estimating the likelihood of toxicity. ${ }^{19}$ The likelihood levels in Derek Nexus in the highest to lowest order are certain, probable, plausible, equivocal, doubted, improbable, and impossible. ${ }^{26}$

4.6. In Silico Prediction of Contilisant Metabolism. To predict metabolism, we used Meteor Nexus ver. 3.1.0 (KB 2018 1.0.0). A knowledge-based approach to rank metabolites based on known metabolic reactions. ${ }^{19}$ To predict the first metabolic step of contilisant, we analyze the Phase I biotransformation pathways, combining two different methods. ${ }^{27}$ A qualitative [absolute reasoning $(\mathrm{AR})$ ] and quantitative [site of metabolism (SOM) scoring] assessment was applied, selecting the matching metabolites. The AR evaluated the likelihood level for biotransformation to occur, and the minimal likelihood level was settled in "plausible," which means that the weight of evidence supports the proposition. ${ }^{26}$ The SOM scoring method uses experimental data for compounds that match the same biotransformation, have similar molecular weights, and are chemically similar around the site of metabolism to contilisant.

4.7. Mutagenic and Carcinogenic Risk Assessment. The International Conference on Harmonisation (ICH) of technical requirements for registration of pharmaceuticals for human use has developed a guideline for the assessment and control of mutagenic impurities to limit potential carcinogenic risk (ICH M7 Assessment and Control of DNA Reactive (Mutagenic) Impurities In Pharmaceuticals To Limit Potential Carcinogenic Risk). This guideline purposes to provide a framework to identify mutagenic alerts with computational toxicology assessment; it has to be performed using two complementary QSAR methodologies. To reach this objective, we have used an in silico prediction system from Lhasa Ltd. (Leeds, UK), Derek Nexus ver. 3.2.0 (expert rule-based methodology) and Sarah Nexus ver. 3.0.0 (statistical-based methodology) to obtain a classification (OECD.2007. Guidance document on the validation of (quantitative) structure-activity relationships [(Q)SAR] models. OECD Environment Health and Safety Publications: Series on Testing and Assessment 69).

\section{ASSOCIATED CONTENT}

\section{(s) Supporting Information}

The Supporting Information is available free of charge at https://pubs.acs.org/doi/10.1021/acschemneuro.0c00489.
Materials and methods; metabolic pathways and clearance predictions in ADMET Predictor; determination of metabolic pathways after $2 \mathrm{~h}$ of incubation with HLMs; endpoints for contilisant after in silico prediction with Derek Nexus v6.0.1; toxicity and mutagenicity risk assessment for contilisant and its predicted metabolites (PDF)

\section{AUTHOR INFORMATION}

\section{Corresponding Author}

Anna Więckowska - Faculty of Pharmacy, Jagiellonian University Medical College, 30-688 Krakow, Poland; (1) orcid.org/0000-0003-2549-170X; Phone: (+48 12) 620 54 50; Email: anna.wieckowska@uj.edu.pl; Fax: (+48 12) 6570262

\section{Authors}

Natalia Szałaj - Faculty of Pharmacy, Jagiellonian University Medical College, 30-688 Krakow, Poland

Izabella Góral - Faculty of Pharmacy, Jagiellonian University Medical College, 30-688 Krakow, Poland

Adam Bucki - Faculty of Pharmacy, Jagiellonian University Medical College, 30-688 Kraków, Poland; 이이.org/00000003-0451-9814

Gniewomir Latacz - Faculty of Pharmacy, Jagiellonian University Medical College, 30-688 Krakow, Poland

Katarzyna Kiec-Kononowicz - Faculty of Pharmacy, Jagiellonian University Medical College, 30-688 Krakow, Poland

Òscar. M. Bautista-Aguilera - Department of Organic Chemistry and Inorganic Chemistry, Alcalá University, 28805 Alcalá de Henares, Madrid, Spain

Alejandro Romero - Department of Pharmacology and Toxicology, Faculty of Veterinary Medicine, Complutense University of Madrid, 28040 Madrid, Spain; (o orcid.org/ 0000-0001-5483-4973

Eva Ramos - Department of Pharmacology and Toxicology, Faculty of Veterinary Medicine, Complutense University of Madrid, 28040 Madrid, Spain; (1) orcid.org/0000-00015791-0687

Javier Egea - Health Research Institute, Clinical Pharmacology Service, University Hospital La Princesa, Autonomous University of Madrid, 28006 Madrid, Spain; Institute Téfilo Hernando for Drug $I+D$, School of Medicine, Autonomous University of Madrid, 28029 Madrid, Spain

Victor Farré Alíns - Health Research Institute, Clinical Pharmacology Service, University Hospital La Princesa, Autonomous University of Madrid, 28006 Madrid, Spain; Institute Téffilo Hernando for Drug $I+D$, School of Medicine, Autonomous University of Madrid, 28029 Madrid, Spain

Águeda González-Rodríguez - Health Research Institute, Clinical Pharmacology Service, University Hospital La Princesa, Autonomous University of Madrid, 28006 Madrid, Spain; Institute Téofilo Hernando for Drug $I+D$, School of Medicine, Autonomous University of Madrid, 28029 Madrid, Spain

Francisco López-Muñoz - Faculty of Health Sciences, University Camilo José Cela, 28692 Villanueva de la Cañada, Madrid, Spain; Neuropsychopharmacology Unit, Hospital 12 de Octubre Research Institute (i+12), 28041 Madrid, Spain

Mourad Chioua - Laboratory of Medicinal Chemistry (IQOG, CSIC), 28006 Madrid, Spain

José Marco-Contelles - Laboratory of Medicinal Chemistry (IQOG, CSIC), 28006 Madrid, Spain 
Complete contact information is available at:

https://pubs.acs.org/10.1021/acschemneuro.0c00489

\section{Author Contributions}

A.W. designed the studies, analyzed the combined data, and revised and prepared the final version of the manuscript. N.S. and I.G. wrote the initial draft of the manuscript. A.B. performed in silico predictions of compounds' metabolism. G.L. performed in vitro studies of metabolic stability, influence on cytochrome P450 (CYPs) isoenzyme activity, and cytotoxicity on HEK-293 and HepG2 cell lines. K.K.-K. supported in vitro studies. Ò.M.B.-A and M.C. carried out the synthesis of PF9601N, ASS234, and contilisant. J.M-C. planned and supervised the synthesis of the target ligands. F.L.-M. supported the research project. A.R. and E.R. performed the in silico toxicological screening of molecules, participated in writing/original draft preparation, and critically revised the manuscript. V.F.A. and A.G.R. performed the toxicological experiments in vitro. J.E. and A.G.R. participated in writing/original draft preparation and critically revised the manuscript.

\section{Notes}

The authors declare no competing financial interest.

\section{ACKNOWLEDGMENTS}

This study received financial support from the National Science Centre Poland (Grant No. 2016/23/D/NZ7/01328). J.M.-C. thanks AEI (Government of Spain) for grants PDI2019-105813RB-C21 and SAF2015-65586-R. J.M.-C. and F.L.M. thank UCJC (Grants UCJC 2020-33 UCJC 2020-03) for support.

\section{REFERENCES}

(1) Poewe, W., Seppi, K., Tanner, C. M., Halliday, G. M., Brundin, P., Volkmann, J., Schrag, A. E., and Lang, A. E. (2017) Parkinson Disease. Nat. Rev. Dis. Primers. 3 (1), 1-21.

(2) Fan, L., Mao, C., Hu, X., Zhang, S., Yang, Z., Hu, Z., Sun, H., Fan, Y., Dong, Y., Yang, J., Shi, C., and Xu, Y. (2020) New Insights Into the Pathogenesis of Alzheimer's Disease. Front. Neurol. 10, 1-12.

(3) Lin, M. T., and Beal, M. F. (2006) Mitochondrial Dysfunction and Oxidative Stress in Neurodegenerative Diseases. Nature 443 (7113), 787-795.

(4) Van Bulck, M., Sierra-Magro, A., Alarcon-Gil, J., Perez-Castillo, A., and Morales-Garcia, J. (2019) Novel Approaches for the Treatment of Alzheimer's and Parkinson's Disease. Int. J. Mol. Sci. 20 (3), 719.

(5) Cavalli, A., Bolognesi, M. L., Minarini, A., Rosini, M., Tumiatti, V., Recanatini, M., and Melchiorre, C. (2008) Multi-Target-Directed Ligands to Combat Neurodegenerative Diseases. J. Med. Chem. 51 (3), 347-72.

(6) Pérez, V., Marco, J. L., Fernández-Álvarez, E., and Unzeta, M. (1999) Relevance of Benzyloxy Group in 2-Indolyl Methylamines in the Selective MAO-B Inhibition. Br. J. Pharmacol. 127 (4), 869-876.

(7) Marco-Contelles, J., Unzeta, M., Bolea, I., Esteban, G., Ramsay, R. R., Romero, A., Martínez-Murillo, R., Carreiras, M. C., and Ismaili, L. (2016) ASS234, as a New Multi-Target Directed Propargylamine for Alzheimer's Disease Therapy. Front. Neurosci. 10 (JUN), 1-7.

(8) Bautista-Aguilera, Ó. M., Hagenow, S., Palomino-Antolin, A., Farré-Alins, V., Ismaili, L., Joffrin, P. L., Jimeno, M. L., Soukup, O., Janočková, J., Kalinowsky, L., Proschak, E., Iriepa, I., Moraleda, I., Schwed, J. S., Romero Martínez, A., López-Muñoz, F., Chioua, M., Egea, J., Ramsay, R. R., Marco-Contelles, J., and Stark, H. (2017) Multitarget-Directed Ligands Combining Cholinesterase and Monoamine Oxidase Inhibition with Histamine H3R Antagonism for
Neurodegenerative Diseases. Angew. Chem., Int. Ed. 56 (41), 1276512769.

(9) Bautista-Aguilera, Ó. M., Budni, J., Mina, F., Medeiros, E. B., Deuther-Conrad, W., Entrena, J. M., Moraleda, I., Iriepa, I., LópezMuñoz, F., and Marco-Contelles, J. (2018) Contilisant, a Tetratarget Small Molecule for Alzheimer's Disease Therapy Combining Cholinesterase, Monoamine Oxidase Inhibition, and H3R Antagonism with S1R Agonism Profile. J. Med. Chem. 61 (15), 6937-6943.

(10) Sanz, E., Quintana, A., Battaglia, V., Toninello, A., Hidalgo, J., Ambrosio, S., Valoti, M., Marco, J. L., Tipton, K. F., and Unzeta, M. (2008) Anti-Apoptotic Effect of Mao-B Inhibitor PF9601N [N-(2Propynyl)-2-(5- Benzyloxy-Indolyl) Methylamine] Is Mediated by P53 Pathway Inhibition in MPP+-Treated SH-SY5Y Human Dopaminergic Cells. J. Neurochem. 105 (6), 2404-2417.

(11) Cutillas, B., Ambrosio, S., and Unzeta, M. (2002) Neuroprotective Effect of the Monoamine Oxidase Inhibitor PF 9601N [N(2-Propynyl)-2-(5-Benzyloxy-Indolyl) Methylamine] on Rat Nigral Neurons after 6-Hydroxydopamine-Striatal Lesion. Neurosci. Lett. 329 (2), $165-168$.

(12) Bolea, I., Gella, A., Monjas, L., Pérez, C., Rodríguez-Franco, M., Marco-Contelles, J., Samadi, A., and Unzeta, M. (2013) Multipotent, Permeable Drug ASS234 Inhibits A $\beta$ Aggregation, Possesses Antioxidant Properties and Protects from $\mathrm{A} \beta$-Induced Apoptosis In Vitro. Curr. Alzheimer Res. 10 (8), 797-808.

(13) Di, L., and Kerns, E. H. Drug-Like Properties: Concepts, Structure Design and Methods from ADME to Toxicity Optimization; Elsevier Inc., 2016.

(14) Więckowska, A., Wichur, T., Godyń, J., Bucki, A., Marcinkowska, M., Siwek, A., Więckowski, K., Zaręba, P., Knez, D., Głuch-Lutwin, M., Kazek, G., Latacz, G., Mika, K., Kołaczkowski, M., Korabecny, J., Soukup, O., Benkova, M., Kieć-Kononowicz, K., Gobec, S., and Malawska, B. (2018) Novel Multitarget-Directed Ligands Aiming at Symptoms and Causes of Alzheimer's Disease. ACS Chem. Neurosci. 9 (5), 1195-1214.

(15) Polasek, T. M., Elliot, D. J., Somogyi, A. A., Gillam, E. M. J., Lewis, B. C., and Miners, J. O. (2006) An Evaluation of Potential Mechanism-Based Inactivation of Human Drug Metabolizing Cytochromes $\mathrm{P} 450$ by Monoamine Oxidase Inhibitors, Including Isoniazid. Br. J. Clin. Pharmacol. 61 (5), 570-584.

(16) Śliwka, L., Wiktorska, K., Suchocki, P., Milczarek, M., Mielczarek, S., Lubelska, K., Cierpiał, T., Łyżwa, P., Kiełbasiński, P., Jaromin, A., Flis, A., and Chilmonczyk, Z. (2016) The Comparison of MTT and CVS Assays for the Assessment of Anticancer Agent Interactions. PLoS One 11 (5), e0155772.

(17) Latacz, G., Lubelska, A., Jastrzębska-Więsek, M., Partyka, A., Marć, M. A., Satała, G., Wilczyńska, D., Kotańska, M., Więcek, M., Kamińska, K., Wesołowska, A., Kieć-Kononowicz, K., and Handzlik, J. (2019) The 1,3,5-Triazine Derivatives as Innovative Chemical Family of 5-HT6 Serotonin Receptor Agents with Therapeutic Perspectives for Cognitive Impairment. Int. J. Mol. Sci. 20 (14), 3420.

(18) Serrano, M. P., Herrero-Labrador, R., Futch, H. S., Serrano, J., Romero, A., Fernandez, A. P., Samadi, A., Unzeta, M., MarcoContelles, J., and Martínez-Murillo, R. (2017) The Proof-of-Concept of ASS234: Peripherally Administered ASS234 Enters the Central Nervous System and Reduces Pathology in a Male Mouse Model of Alzheimer Disease. J. Neuropsychiatry Clin. Neurosci. 42 (1), 59-69.

(19) Marchant, C. A., Briggs, K. A., and Long, A. (2008) In Silico Tools for Sharing Data and Knowledge on Toxicity and Metabolism: Derek for Windows, Meteor, and Vitic. Toxicol. Mech. Methods 18 (23), 177-187.

(20) Crumb, W., and Cavero, I. (1999) QT Interval Prolongation by Non-Cardiovascular Drugs: Issues and Solutions for Novel Drug Development. Pharm. Sci. Technol. Today 2 (7), 270-280.

(21) OECD (2007) Guidance Document on the Validation of (Quantitative) Structure-Activity Relationship [(Q)Sar] Models. Transport 2, 1-154.

(22) Lopez, M. F., Marco, C. J. L., Stark, H., Hagenow, S., and Ramsay, R. R. (2020) Nuevos compuestos con capacidad antioxidante que combinan la inhibición de las enzimas monoaminoxidasas y 
colinesterasas y la interacción con el receptor de histamina 3, su procedimiento de obtención y composiciones farmacéuticas que los contienen. ES2701954A1. Provided by https://patents.google.com/ patent/ES2701954A1/es (accessed 2020-07-10).

(23) van Meerloo, J., Kaspers, G. J. L., and Cloos, J. (2011) Cell Sensitivity Assays: The MTT Assay. Methods Mol. Biol. 731 (88), 237-245.

(24) Gonzalez-Rodriguez, A., Clampit, J. E., Escribano, O., Benito, M., Rondinone, C. M., and Valverde, A. M. (2007) Developmental Switch from Prolonged Insulin Action to Increased Insulin Sensitivity in Protein Tyrosine Phosphatase 1B-Deficient Hepatocytes. Endocrinology 148 (2), 594-608.

(25) Feoktistova, M., Geserick, P., and Leverkus, M. (2016) Crystal Violet Assay for Determining Viability of Cultured Cells. Cold Spring Harb. Protoc. 2016 (4), 343-346.

(26) Judson, P. N., Stalford, S. A., and Vessey, J. (2013) Assessing Confidence in Predictions Made by Knowledge-Based Systems. Toxicol. Res. 2 (1), 70-79.

(27) al Sharif, M., Alov, P., Vitcheva, V., Diukendjieva, A., Mori, M., Botta, B., Tsakovska, I., and Pajeva, I. (2017) Natural Modulators of Nonalcoholic Fatty Liver Disease: Mode of Action Analysis and in Silico ADME-Tox Prediction. Toxicol. Appl. Pharmacol. 337, 45-66. 